\title{
Optimization design on reliability of three-state electrical redundant system $^{1}$
}

\author{
Zhiliang WU ${ }^{1, a}$, Yubin $Y^{2, b}$, Dan WANG ${ }^{3, c}$ \\ ${ }^{1}$ Marine Eng. College, Dalian Maritime Univ., Dalian, 116026, China \\ ${ }^{2}$ Marine Eng. College, Dalian Maritime Univ., Dalian, 116026, China \\ ${ }^{3}$ Marine Eng. College, Dalian Maritime Univ., Dalian, 116026, China \\ aemail: wzldmu@126.com
}

Key wards: three-state; redundancy; parallel; reliability; electrical system

\begin{abstract}
The methods of reliability analysis for parallel-redundant system are different from the traditional two-state system, according to multi-state reliability theory, the design of the three-state electrical parallel-redundant system was optimized, and a mathematical model of the reliable degree for the system was built. The formula of reliable degree was given by an example with usual three-state electrical parallel-redundant system. Researches indicated that it couldn't increase the reliability of the system even adopting the three-state electrical parallel-redundant system with more parallel-redundant branches because of the existence of short circuit failure. On the contrary, it would decrease the reliable degree of system with excessive branches. It differs from two-state system that the optimal redundancy is only 2 for the multi-branch redundancy design with auxiliary contacts of relay which often used in three-state electrical parallel-redundant system. The research results have universality, they are appropriate for electrical parallel-redundant system and the general three-state parallel-redundant system.
\end{abstract}

\section{Introduction}

In the design of electrical system, the reliability of system's Achilles heel has been greatly improved by adopting redundancy design, thus the reliability of system has also been improved. Due to the diversity of redundancy design, the optimization design of redundancy has become the key to the reliability design of electrical system ${ }^{[1]}$.

To achieve the goal of reliability, redundancy technique is usually adopted as one of the important methods in the field of electric engineering and automation ${ }^{[2]}$. Traditional reliability analysis methods for the redundant system include Reliability Block Diagram, Fault Tree Analysis, Fault Modes and Effects Analysis and so on, which divide the system and elements into two states, namely "normal" and "failure". The system is modeled and analyzed by adopting binary logic. As for electrical system, its working status are multiple because of the diversity of failure mechanism for elements, so it is unreasonable to divide system and element into two states. The simple binary hypothesis causes the great difference between the system mathematical and the actual condition. Two-state reliability theory can't describe the complex failure process accurately between system and elements, which will bring larger deviation and can't meet the needs of engineering.

For example, the main failure modes for auxiliary contacts of the relay are contacts open-circuit failure, contacts short-circuit failure and so on; the main failure modes for diodes are open-circuit failure, short-circuit failure and parameter drifts and so on $^{[3]}$. If such elements or systems are designed and analyzed in the aspect of reliability redundancy as two-state system, it will lead to inaccuracy even error. In order to improve the calculation accuracy of redundancy system and determine the optimal redundancy, electrical control system should be regarded as multi-state system to process reliability modeling, analysis and design ${ }^{[4]}$.

\footnotetext{
${ }^{1}$ Foundation project: funding project of the national nature science foundation project (61273137)

About the author: Wu zhiliang(1963-), Man, Dalian, Liaoning, Doctor, Professor, engaged in teaching and research on marine electrical automation system.E-mail: wzldmu@126.com 
The multi-state reliability can reveal the potential failure mechanism of complex system effectively and solve the modern engineering reliability problems of large complex equipments and systems, which has become the difficult and hot spot in the academia and industry ${ }^{[5]}$.

More and more people pay attention to the multi-state reliability, which is still evolving and improving. It has been one of the hot topics in the field of reliability, and a lot of researches on the multi-state reliability theory have been carried by some scholars at home and abroad.

The article takes three-state electrical parallel redundancy system for example, establishing reliability mathematical model, and the design of three-state electrical parallel redundant system is optimized.

\section{The reliability analysis of three-state parallel redundancy system}

In order to improve the reliability of the system, parallel redundancy system is applied most widely in electrical system. The article takes auxiliary contacts of the relay parallel redundancy design in electrical system for example, to analyze the reliability of three-state electrical parallel redundancy system. The diagram of reliability of auxiliary contacts of the relay parallel redundancy system is shown in figure 1.

The auxiliary contacts of the relay is multimode, including normal state, open failure state and short failure state, so the system composed of auxiliary contacts of the relay is three-states parallel redundancy system.

If: $x_{i}$ represents the normal state of auxiliary contacts of the relay;

$\bar{x}_{i \mathrm{o}}$ represents the open failure state of auxiliary contacts of the relay;

$\bar{x}_{i s}$ represents the short failure state of auxiliary contacts of the relay; $(\mathrm{i}=1 \sim \mathrm{n})$

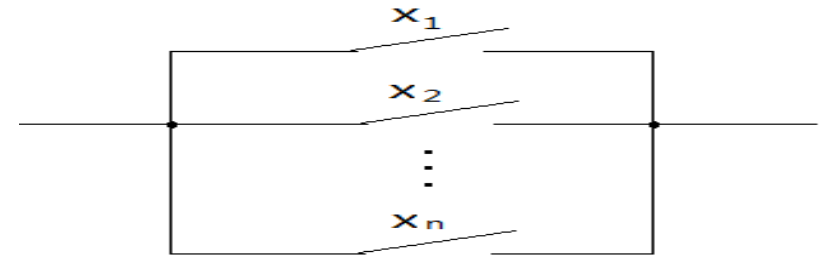

The probability of them is:

Fig 1 the diagram of system reliability

$$
\begin{gathered}
P\left(x_{i}\right)=P_{i} \\
P\left(\bar{x}_{i 0}\right)=q_{i 0} \\
P\left(\bar{x}_{i \mathrm{~s}}\right)=q_{i \mathrm{~s}} \\
\text { and } P_{i}+q_{i 0}+q_{i \mathrm{~s}}=1
\end{gathered}
$$

If $t=0$, namely each element is brand new, the longevity of the elements of the system obey the exponential distribution, and the failure rate is constant. There are two kinds of failure states, namely open failure mode and short failure mode, and the two failure mode is mutually exclusive and separate.

Definition: in the parallel system which is consist of $n$ branches, all the branches appear the open-circuit failure, call the system open-circuit failure. If there is at least one branch appear short-circuit failure, call the system short-circuit failure.

First to be considered is the two-branch electrical parallel-redundant system which consist of two relay auxiliary contacts $\mathrm{x} 1$ and x2, only when this two contacts won't appear open-circuit failure in the meantime or anyone won't appear short-circuit failure, then the system is reliable.

So the probability of reliable degree for two-branch parallel-redundant system:

$$
\begin{aligned}
R & =1-P\left[\bar{x}_{1 \mathrm{o}} \bar{x}_{2 \mathrm{o}}+\bar{x}_{1 \mathrm{~s}}+\bar{x}_{2 \mathrm{~s}}\right] \\
& =1-\left\{\left[P\left[\bar{x}_{1 \mathrm{o}} \bar{x}_{2 \mathrm{o}}\right]+P\left(\bar{x}_{1 \mathrm{~s}}+\bar{x}_{2 \mathrm{~s}}\right)-P\left[\left(\bar{x}_{1 \mathrm{o}} \bar{x}_{2 \mathrm{o}}\right)\left(\bar{x}_{1 \mathrm{~s}}+\bar{x}_{2 \mathrm{~s}}\right)\right]\right\}\right. \\
& =1-P\left[\bar{x}_{1 \mathrm{o}} \bar{x}_{2 \mathrm{o}}\right]-P\left(\bar{x}_{1 \mathrm{~s}}+\bar{x}_{2 \mathrm{~s}}\right)+P\left[\left(\bar{x}_{1 \mathrm{o}} \bar{x}_{2 \mathrm{o}}\right)\left(\bar{x}_{1 \mathrm{~s}}+\bar{x}_{2 \mathrm{~s}}\right)\right]
\end{aligned}
$$

Because the relay auxiliary contacts can't appear two failure mode at the same time, the probability of final part above the formula is zero, that's to say:

$$
P\left[\left(\bar{x}_{1 \mathrm{o}} \bar{x}_{2 \mathrm{o}}\right)\left(\bar{x}_{1 \mathrm{~s}}+\bar{x}_{2 \mathrm{~s}}\right)\right]=0
$$

Since the failure modes are independent from each other, then: 


$$
\begin{aligned}
R & =1-P\left(\bar{x}_{1 \mathrm{o}} \bar{x}_{2 \mathrm{o}}\right)-\mathrm{P}\left(\bar{x}_{1 \mathrm{~s}}+\bar{x}_{2 \mathrm{~s}}\right) \\
& =1-P\left(\bar{x}_{1 \mathrm{o}}\right) P\left(\bar{x}_{2 \mathrm{o}}\right)-P\left(\bar{x}_{1 \mathrm{~s}}\right)-P\left(\bar{x}_{2 \mathrm{~s}}\right)+P\left(\bar{x}_{1 \mathrm{~s}}\right) P\left(\bar{x}_{2 \mathrm{~s}}\right) \\
& =1-q_{1 \mathrm{o}} q_{2 \mathrm{o}}-q_{1 \mathrm{~s}}-q_{2 \mathrm{~s}}+q_{1 \mathrm{~s}} q_{2 \mathrm{~s}}
\end{aligned}
$$

Suppose the failure probability of auxiliary are equal, and

$$
\begin{aligned}
& q_{1 \mathrm{o}}=q_{2 \mathrm{o}}=q_{\mathrm{o}} \\
& q_{1 \mathrm{~s}}=q_{2 \mathrm{~s}}=\mathrm{q}_{\mathrm{s}}
\end{aligned}
$$

So the probability of reliable degree

For further deduction:

$$
R=\left(1-q_{\mathrm{s}}\right)^{2}-q_{\mathrm{o}}^{2}
$$

The probability of reliable degree for n-branch parallel system which consists of $n$ three-state relay auxiliary contacts:

$$
R=\left(1-q_{\mathrm{s}}\right)^{n}-q_{\mathrm{o}}^{n}
$$

In order to calculate the optimal branch number $n$ when the probability of reliable degree $\mathrm{R}$ is maximum, suppose:

So:

$$
\frac{\partial R}{\partial n}=0
$$

$$
n=\frac{\ln \left\{\left[\ln \left(1-q_{\mathrm{s}}\right)\right] / \ln q_{\mathrm{o}}\right\}}{\ln \left[q_{\mathrm{o}} /\left(1-\mathrm{q}_{\mathrm{s}}\right)\right]}
$$

Usually $n=\left\{\begin{array}{l}{[n]+1(n \text { is not a integer, }[n] \text { is the integral part of real number })} \\ n\end{array}\right.$

\section{Applied computational example}

For the two-state electrical system, we can use mathematical induction to prove that: the reliable degree of parallel system which is composed of the same sub-system is more than the series one. And with the increase of the component of parallel system, the reliable degree shows the trend of increasing. Therefore, the design of multi-branch parallel-redundant system usually appears in traditional electrical design, for example, the electromagnetic operate closing circuit of DW95 air circuit breaker adopts 3 couples of normally open relay auxiliary contacts to realize the parallel-redundant design, the block diagram of reliability for this system as shown in the figure 2 .

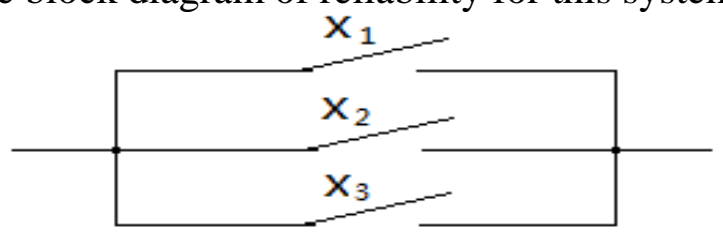

Fig. 2 the block diagram of system reliability

According to the document 3 , the universal failure rate of relay $\lambda=1.1 \times 10^{-6} /$ hour , the open-circuit failure rate of relay auxiliary contacts $\lambda_{o}=0.484 \times 10^{-6} /$ hour , the short-circuit failure rate of relay auxiliary contacts $\lambda s=0.44 \times 10^{-6} /$ hour .

The reliability analysis of routine two-state method:

Figure 2 shows the typical three-branch parallel system, the reliable degree:

$$
R=3 e^{-\lambda t}-3 e^{-2 \lambda t}+e^{-3 \lambda t}
$$

Suppose the work time $\mathrm{t}=1$ year $=8760 \mathrm{~h}$, then the reliable degree of system:

$$
R=0.99999
$$

The reliability analysis of the three-state method:

The reliable degree of the system:

$$
R=\left(1-q_{\mathrm{s}}\right)^{3}-q_{\mathrm{o}}^{3}
$$

In this formula: the short-circuit failure probability of relay auxiliary contacts:

$$
q_{\mathrm{s}}=1-\mathrm{e}^{-\lambda_{\mathrm{s}} t}
$$

The open-circuit failure probability of relay auxiliary contacts: 


$$
q_{\mathrm{o}}=1-\mathrm{e}^{-\lambda_{\mathrm{o}} t}
$$

Suppose the work time $t=1$ year=8760h, substitute it into formula (3-1), then the reliable degree of system:

$$
R=0.9886
$$

Thus is can be seen that the calculating result of reliable degree differs a lot for the analysis of routine two-state method and the three-state method. When analyzing practical engineering applications, because of the incomplete cognition to the system in traditional theories, the model of parallel-redundant system which based on the reliability analysis of routine two-state method is usually not accurate, directly cause some misunderstanding for the design of multi-branch parallel system in practical engineering applications. Since practical systems usually exist multiple failure mode, and for most systems, especially for the electrical systems that require enough high reliability, it is required to build a more accurate mathematical reliability model of multi-state parallel-redundant system, and be able to confirm the optimal parallel redundancy of the design for multi-branch parallel-redundant system to satisfy the demands for practical engineering applications.

The confirm of optimal parallel redundancy for multi-branch parallel-redundant system:

Suppose the work time $t=1$ year $=8760 h$, substitute it into formula (2-2), (2-3), then we can get a result:

$$
\mathrm{n}=2
$$

That's to say when the number of branches $n=2$ for three-state parallel-redundant system, the value of reliable degree $\mathrm{R}$ is maximum.

Suppose $n=2$, the work time $t=1$ year $=8760$ h, substitute these two parameters into formula (2-1), the reliable degree of system:

$$
\begin{aligned}
R= & \left(1-q_{\mathrm{s}}\right)^{2}-q_{\mathrm{o}}^{2} \\
= & 0.9924
\end{aligned}
$$

So the conclusions can be gotten from this example: because of the existence of short-circuit failure, it couldn't increase the reliability of the system even adopting the three-state electrical parallel redundant system that consists of more parallel redundant branches, on the contrary, it would decrease the reliable degree of parallel redundant system with excessive parallel branches. It differs from two-state system that the optimal redundancy is only 2 for the multi-branch redundancy design with auxiliary contacts of relay which often used in three-state electrical parallel redundant system.

\section{Conclusion}

In the traditional analysis and design for the reliability of electrical system, electrical parallel-redundant system is processed and analyzed as two-state system, that's to say the system and all components that consist the system are considered to have only formal and failure two-state, but the most components of electrical system have multiple states in practical system, therefore the electrical system which is made up of multi-state components also has multiple failure state, it is called multi-state system.

This passage has built a mathematical model of reliable degree for three-state parallel-redundant system, and optimized the design of the system. The example has given the reliable degree of usual three-state electrical parallel-redundant system and optimized the design.

\section{References}

[1] Zhao Zhicao, Song Baowei, et al. Optimization of Real-time Reliability Redundancy in Complex System[J]. Journal of Northwestern Polytechnical University, 2013, 31(1): 134-138

[2] Cao Jinhua, Cheng Kan. Mathematical theory of reliability [M]. Beijing: Higher Education Press, 2012. 
[3] Wu Zhiliang. Reliability engineering and Application on electrical system of ships and ports[M]. Dalian: Dalian Maritime University Press, 2006.

[4] R.Billinton,Y.Gao. Adequacy assessment of composite Power generation and transmission systems with wind energy[J]. International Journal of Reliability and Safety, 2008, 2(1-2):79-98

[5] Pang Yu, Huang Hongzhong, et al. Possibility Theory Based Multi-State System Reliability Analysis[J]. Journal of Xi'an Jiaotong University, 2013， 47(3): 75-79 